\title{
Nonalcoholic Fatty Liver Disease: Epidemiology, Liver Transplantation Trends and Outcomes, and Risk of Recurrent Disease in the Graft
}

\author{
Andy Liu* ${ }^{1}$, Artin Galoosian ${ }^{1}$, Dharmesh Kaswala ${ }^{2}$, Andrew A. Li ${ }^{3}$, Chiranjeevi Gadiparthi ${ }^{4}$, \\ George Cholankeril ${ }^{3}$, Donghee $\mathrm{Kim}^{3}$ and Aijaz Ahmed ${ }^{3}$ \\ ${ }^{1}$ California Pacific Medical Center, Department of Medicine, San Francisco, CA, USA; ${ }^{2}$ California Pacific Medical Center, Division of \\ Gastroenterology and Hepatology, San Francisco, CA, USA; ${ }^{3}$ Stanford University School of Medicine, Division of Gastroenterology \\ and Hepatology, Stanford, CA, USA; ${ }^{4}$ University of Tennessee Health Science Center, Division of Gastroenterology and \\ Hepatology, Memphis, TN, USA
}

\begin{abstract}
In parallel with the rising prevalence of metabolic syndrome globally, nonalcoholic fatty liver (NAFL) disease is the most common chronic liver disease in Western countries and nonalcoholic steatohepatitis (NASH) has become increasingly associated with hepatocellular carcinoma. Recent studies have identified NASH as the most rapidly growing indication for liver transplantation (LT). As a hepatic manifestation of the metabolic syndrome, NAFL disease can be histologically divided into NAFL and NASH. NAFL is considered a benign condition, with histological changes of hepatocyte steatosis but without evidence of hepatocellular injury or fibrosis. This is distinct from $\mathrm{NASH}$, which is characterized by hepatocyte ballooning and inflammation, and which can progress to fibrosis and cirrhosis, hepatocellular carcinoma, and liver failure. As for any other end-stage liver disease, LT is a curative option for NASH after the onset of decompensated cirrhosis or hepatocellular carcinoma. Although some studies have suggested increased rates of sepsis and cardiovascular complications in the immediate postoperative period, the long-term posttransplant survival of NASH cases is similar to other indications for LT. Recurrence of NAFL following LT is common and can be challenging, although recurrence rates of NASH are lower. The persistence or progression of metabolic syndrome components after LT are likely responsible for NASH recurrence in transplanted liver. Therefore, while maintaining access to LT is important, concerted effort to address the modifiable risk factors and develop effective screening strategies to identify early stages of disease are paramount to effectively tackle this growing epidemic.
\end{abstract}

Citation of this article: Liu A, Galoosian A, Kaswala D, Li AA, Gadiparthi C, Cholankeril G, et al. Nonalcoholic fatty liver

Keywords: Nonalcoholic fatty liver disease; Nonalcoholic steatohepatitis; Liver transplantation; Epidemiology; Trends.

Abbreviations: AASLD, American Association for the Study of Liver Disease ALD, alcoholic liver disease; BMI, body mass Index; CI, confidence interval; ESLD, end-stage liver disease; HCC, hepatocellular carcinoma; HCV, hepatitis C virus; HR, hazard ratio; HTN, hypertension; LT, liver transplantation; NAFLD, nonalcoholic fatty liver disease; NASH, nonalcoholic steatohepatitis; NHANES, National Health and Nutrition Examination Survey; OPTN, Organ Procurement and Transplantation Network; OR, odds ratio; T2DM, type 2 diabetes mellitus; UNOS, United Network for Organ Sharing.

Received: 15 February 2018; Revised: 15 May 2018; Accepted: 31 May 2018

*Correspondence to: Andy Liu, Department of Medicine, California Pacific Medical Center 2351 Clay Street, Suite 380, San Francisco, CA 94110, USA. Tel: +1-415-600-3954, Fax: +1-415-775-7437, E-mail: andyeliu@gmail.com disease: Epidemiology, liver transplantation trends and outcomes, and risk of recurrent disease in the graft. J Clin Transl Hepatol 2018;6(4):420-424. doi: 10.14218/JCTH.2018.00010.

\section{Introduction}

As the prevalence of obesity increases worldwide, related complications including dyslipidemia, insulin resistance and metabolic syndrome are increasing as well. ${ }^{1}$ Insulin resistance and metabolic alterations are closely associated with nonalcoholic fatty liver disease (NAFLD). As such, NAFLD is sometimes known as the "hepatic manifestation" of the metabolic syndrome and mirrors the rising rates of hyperlipidemia, obesity, insulin resistance, endothelial dysfunction, and/ or type 2 diabetes mellitus (T2DM) ${ }^{2,3}$ Because of the association with metabolic syndrome, NAFLD is currently one of the most common chronic liver diseases in Western countries. NAFLD is also emerging as a major cause of hepatocellular carcinoma (HCC), rising at a rate higher than any other etiology of liver disease. ${ }^{1,4-6}$

NAFLD represents a spectrum of diseases, ranging from fatty liver without inflammation to nonalcoholic steatohepatitis (NASH) and cirrhosis. Per the latest definition by the American Association for the Study of Liver Diseases (commonly known as AASLD), NAFLD entails two components: 1) evidence of hepatic steatosis by imaging or histology; and 2) exclusion of other etiologies for hepatic steatosis including significant alcohol consumption, medication adverse effect, and/or hereditary disorders. ${ }^{7}$ Histologically, NAFLD can be further subdivided into nonalcoholic fatty liver (NAFL) or NASH. NAFL is defined as the presence of greater than or equal to $5 \%$ hepatic steatosis without hepatocellular injury in the form of hepatocyte ballooning or fibrosis. $\mathrm{NASH}$, on the other hand, entails greater than or equal to $5 \%$ hepatic steatosis with inflammation and hepatocyte injury with or without fibrosis. Further progression of disease into cirrhosis is termed NASH cirrhosis and can result in HCC and liver failure.

\section{Pathogenesis}

While hypertriglyceridemia and insulin resistance are associated hallmarks of NAFLD, the pathogenesis of NAFLD is not 
fully elucidated. Questions remain regarding the factors underlying inflammation and disease progression. The development of steatosis in the liver is the first step, which is associated with a state of chronic hepatic inflammation. ${ }^{8}$ In vivo studies of mice have shown that a high fat diet leads to an over-expression of proinflammatory markers such as nuclear factor-kappa B as well as pro-inflammatory cytokines such as TNF- $\alpha$, IL-6, and IL-1 $\beta .{ }^{9-11}$ Inflammation from myeloid cells, such as Kupffer cells, may also play a large part in obesity-induced insulin resistance. ${ }^{11}$ Additionally, studies have also demonstrated genetic modifiers for development of NAFLD.

A genome-wide association study found that the rs738409[G] allele in PNPLA3 was strongly associated with increased hepatic fat levels and hepatic inflammation. ${ }^{12}$ This allele was most common in Hispanics, and allelic homozygosity was associated with hepatic fat levels more than twice as high as in noncarriers. Another study in an Eastern European population and published in March 2017 found PNPLA3 rs738409 to be associated with increased risk of developing fibrosis (odds ratio $(O R)=1.65, p=0.001$ ) and cirrhosis $(\mathrm{OR}=1.92, p<0.005) \cdot{ }^{13}$ Recent studies have confirmed the interplay between genetic predispositions and environmental factors. A study published in June 2017 showed that the three sequence variants encoding PNPLA3-I148M, TM6SF2-E167K and GCKR-P446L are significantly associated with NAFLD. ${ }^{14}$

\section{Epidemiology}

The true prevalence of NAFLD is unknown. Most of the available data is from epidemiological estimates. Younossi et al., ${ }^{1}$ using a meta-analysis of 86 studies with a combined sample size of $8,515,431$ from 22 countries, estimated that the global NAFLD prevalence is $25 \%$ ( $95 \%$ confidence interval (CI): $22-29 \% ; p<0.05)$. The pooled data showed that NAFLD is highly prevalent in all continents, but the highest rates have been reported from South America (31\%) and the Middle East $(32 \%)$, followed by Asia (27\%), the USA $(25.8 \%)$, and Europe (23\%). ${ }^{1}$ Prevalence of NASH is estimated to range from $1.5 \%$ to $6 \% \cdot{ }^{1,15-17}$ In their studies, pooled NASH prevalence amongst NAFLD patients undergoing biopsy was $59.1 \%\left(95 \% \mathrm{CI}\right.$ : 55.16-94.28). ${ }^{1}$ However, the estimates of NASH prevalence and fibrosis progression rates among individuals with NAFLD are limited by the largely retrospective methodology used in numerous studies and the limitations in the screening guideline consensus. ${ }^{1,18-23}$

Most patients are diagnosed with NAFLD between the fourth and fifth decade of life. ${ }^{24}$ Studies have also reported mixed results regarding sex-related predominance, with earlier studies suggesting a female predominance $(65-83 \%){ }^{24}$ However, in a 2018 study using a cross-sectional survey based on the National Health and Nutrition Examination Survey (commonly known as NHANES) III, sex distribution of NAFLD varied by age group. ${ }^{23}$ The lowest male-to-female prevalence (0.94) was seen in individuals less than 30 years old, while the highest ratio (1.31) was seen in individuals aged 40-49 years. The prevalence of NAFLD also has significant ethnic variations. A study of hepatic triglyceride levels of 2,287 individuals from a USA population-based sample found that the highest rate of NAFLD was seen in Hispanics (45\%), followed by non-Hispanic whites (33\%); the lowest prevalence was seen in African Americans (24\%). ${ }^{25}$ The higher rates of NAFLD in Hispanics were associated with correspondingly higher rates of obesity and insulin resistance in this group. These observed differences are likely multifactorial and related to a combination of genetic and environmental factors. 26,27

Using data from the NHANES III, the incidence of NAFLD in 2015 was projected to be around 3.44 million. ${ }^{23}$ However, that annual incidence is estimated to decrease by more than $30 \%$ in the next decade, which some authors attribute to a decrease in the overall incidence of obesity in the general population. ${ }^{23,28}$ Although a decrease in the incidence is expected to occur, the prevalence will continue to rise. ${ }^{17,23}$ Due to the increased prevalence of NAFL, the prevalence of $\mathrm{NASH}$ is expected to increase as well. ${ }^{1,4,23}$ Estes et al. ${ }^{23}$ estimate that the proportion of NAFL cases that progress to NASH will increase from $20 \%$ to $27 \%$ by 2030 . Similarly, incident decompensated cirrhosis is predicted to increase by $168 \%$, from 39,230 cases annually in 2015 to 105,430 cases in 2030. The corresponding burden of NASH cirrhosis on liver transplantation (LT) is expected to increase by $59 \% .^{23}$ Numerous recent studies have reported that NASH-related cirrhosis is the most rapidly growing indication for LT in the USA. ${ }^{29-32}$

\section{Changing epidemiology landscape of chronic liver diseases and LT trends}

The prevalence of NAFLD continues to increase, and it is expected that NASH-related end-stage liver disease (ESLD) will become the leading indication for LT over the next several decades. ${ }^{1,4,29,30,33}$ Studies have demonstrated a disproportionate increase in NASH-related cirrhosis as an indication for LT relative to other etiologies. ${ }^{31,33}$ In 2017, Cholankeril et al., ${ }^{31}$ using data from the United Network for Organ Sharing and Organ Procurement and Transplantation Network (commonly known as UNOS/OPTN) database, reported a $162 \%$ increase in LT secondary to NASH from 2003 to 2014, while LT due to alcoholic liver disease (ALD) and hepatitis C virus (HCV) only increased by $54 \%$ and $33 \%$ respectively. As the burden of NASH-related ESLD on the wait-list continues to rapidly grow, the risk of wait-list removal secondary to death or progression of comorbidities increases. ${ }^{34}$

According to Wong et al., ${ }^{30}$ from 2004-2013, NASHrelated disease was the second leading etiology of liver disease among adults on the LT wait-list in the USA. During this period, the number of new wait-list listings increased by $170 \%$. From 2007 to 2015 , the absolute number of NASHrelated listings on the UNOS registry increased each year. ${ }^{35}$ In contrast, during the same period, new waitlist registrations for ALD increased $45 \%$ and HCV by a mere $14 \%$, whereas HCV/ALD decreased by $9 \%$, reflecting the changing epidemiological landscape for LT. $^{30}$ Importantly, since the advent of highly effective direct-acting antiviral agents in 2013, HCV disease burden and need for LT have decreased dramatically. Wong et al. ${ }^{30}$ also demonstrated worse outcomes for 90-day wait-list survival for NASH, when compared to HCV, HCV/ALD, and ALD.

Despite higher wait-list mortality in patients with ALD, when adjusted for sex, age, race/ethnicity, presence of T2DM, model for end-stage liver disease score, ascites, and concurrent HCC using multivariate analysis modeling, ALD patients demonstrated increased 90-day wait-list survival compared to those with NASH (OR $=0.77$, 95\%CI: $0.67-$ $0.89, p<0.001)$. Wait-list survival at 1 -year was similar for ALD patients showing superior survival when compared to 
NASH patients $(\mathrm{OR}=0.74,95 \% \mathrm{CI}: 0.66-0.83, p<0.001){ }^{30}$ Furthermore, among NASH patients awaiting LT, a 2017 study found that morbid obesity and T2DM are associated with higher rates of wait-list removal or mortality. ${ }^{36}$ When comparing probabilities of receiving a liver transplant, patients with NASH were less likely to undergo LT. ${ }^{30}$ Compared to NASH, patients with HCV, ALD, and HCV/ALD were more likely to undergo LT within 90 days and 1 year of being on the waitlist registry. Additionally, a 2017 study by Stine et al. ${ }^{37}$ showed that those with high-risk NASH (as defined by age $>60$ years, body mass index $(B M I)>30 \mathrm{~kg} / \mathrm{m}^{2}$, hypertension (HTN), and T2DM), were at a higher risk of developing pretransplant portal vein thrombosis compared to non-NASH patients $(\mathrm{OR}=2.11,95 \% \mathrm{CI}: 1.60-2.76)$.

\section{Outcomes of LT for NASH-related cirrhosis}

In patients undergoing LT for NASH-related cirrhosis, longterm mortality outcomes are similar to those who undergo LT for other etiologies, such as ALD and HCV. ${ }^{33,38,39}$ Reported 1 -year posttransplant survival is approximately $79-90 \%$, 3 -year survival is $82-83 \%$, and 5 -year survival is $72-$ $78 \% .{ }^{31,33,38,40}$ Malik et al. ${ }^{40}$ performed a subanalysis of patients who died within the first year of transplantation and found that these patients were more likely to be of age $\geq 60$ years, BMI $\geq 30 \mathrm{~kg} / \mathrm{m}^{2}$, and have pretransplant HTN and T2DM. In this higher-risk group, the 30-day mortality and 1 -year mortality were $31 \%$ and $50 \%$, respectively. Cause of death in NASH patients posttransplant was primarily infection $(57.1 \%)$, statistically significantly higher when compared to other indications for $\mathrm{LT}^{40}$

A meta-analysis in 2014 by Wang et al. ${ }^{41}$ also found that patients with NASH had a greater risk of death from cardiovascular complications $(\mathrm{OR}=1.65,95 \% \mathrm{CI}$ : 1.01-2.70, $p=0.05)$. The same risk factors of metabolic syndrome that predispose patients to NAFLD, including obesity, T2DM, hyperlipidemia and HTN, are also risk factors for developing coronary artery disease. It is also well established that these risk factors are associated with a higher incidence of cardiac events following any type of surgery and that the risk of infection is increased in patients with T2DM. ${ }^{42-44}$ Despite these known risk factors, recent studies have shown that survival rates and long-term outcomes are similar among ALD, HCV, and NASH-related cirrhosis. ${ }^{41}$ In their meta-analysis including 717 patients with $\mathrm{NASH}$ and 3,520 without $\mathrm{NASH}$, Wang et $a .^{41}$ also found that patient mortality after transplant was similar between NASH recipients and control subjects. The analysis was based on the survival rates for the primary indication of NASH-related ESLD versus nonNASH-related ESLD (including ALD and HCV), at years 1, 3, and 5 posttransplantation (OR at 1 -year postLT $=0.77,95 \% \mathrm{CI}$ : 0.59-1.00, $p=0.05$; OR at 3 -years postLT $=0.97,95 \% \mathrm{CI}: 0.67-1.40$, $p=0.86 ;$ OR at 5 -years postLT $=1.09,95 \% \mathrm{CI}: 0.77-1.56$, $p=0.63) .41$

In addition, in a retrospective cohort study in 2017 comparing postLT outcomes in $\mathrm{NASH}, \mathrm{HCV}$, and ALD, patients with NASH had a significantly higher posttransplant survival compared to patients with $\mathrm{HCV}$ (hazard ratio $(\mathrm{HR})=0.75$, $95 \% \mathrm{CI}: 0.71-0.79, p<0.001)$ and ALD $(\mathrm{HR}=0.80,95 \% \mathrm{CI}$ : $0.76-0.84, p<0.001){ }^{31}$

\section{Recurrence of NAFLD after LT}

As early as 1997 , recurrence of NASH in patients undergoing LT due to NASH cirrhosis has been reported. ${ }^{45}$ Generally following LT, patients have a known propensity for weight gain and obesity. In one study of BMI after LT, among 320 patients who were not obese prior to transplantation, $21.6 \%$ became obese within 2 years after transplantation. 46 The prevalence of obesity and metabolic syndrome components, including increased insulin resistance following $\mathrm{LT}$, place patients at risk for recurrent NAFL and NASH, as well as de novo NAFLD. Multiple factors have been reported to cause recurrence of $\mathrm{NASH}$, including obesity, use of steroids, insulin use, HTN, and hypertriglyceridemia. Contos et al. ${ }^{47}$ studied patients with cirrhosis due to NASH undergoing LT and found a mean BMI increase of 2.2 at a mean follow-up of 4.5 years. In that study, 12 of $28(42.9 \%)$ patients developed recurrent steatosis. Only a change in BMI $>3$ after LT was associated with recurrent steatosis ( $\mathrm{OR}=1.5, p<0.05)$ on routine surveillance biopsy. ${ }^{47}$ Similar recurrence rates for NASH have been described.

A single-center study by Bhati et al. ${ }^{48}$ analyzed all patients who received a LT between 1995 and 2013 due to NASH cirrhosis or cryptogenic cirrhosis where NASH was suspected. Of the 34 patients who underwent liver biopsy posttransplant, $88.2 \%$ showed NAFLD recurrence, while $41.2 \%$ showed NASH recurrence. Median time from the LT was 47 months. Evaluation of 56 patients after LT by transient elastography found recurrence at a similar rate of $87.5 \%$. Median time was 75 months. Further stratification of patients with recurrent NAFLD on biopsy found that although T2DM prevalence between patients with recurrence and those without recurrence were similar, fasting glucose in those with recurrence of NAFLD were significantly higher than in those without disease recurrence $(169 \pm 81 \mathrm{mg} / \mathrm{dL}$ vs. $98 \pm 3 \mathrm{mg} / \mathrm{dL}$, $p<0.01)$. In the patients followed by transient elastography, T2DM prevalence was higher in patients with recurrent NAFLD ( $81 \%$ vs. $51 \%, p=0.08$ ). In addition, serum triglyceride levels were higher in patients with recurrent NAFLD compared to those without recurrence. The recurrence rates in this cohort may have been higher relative to other studies of NAFLD recurrence in patients undergoing LT given the higher prevalence of metabolic syndrome in these patients.

Bhagat et al. ${ }^{49}$ reported similar high rates of NASH recurrence, with $33 \%$ of patients transplanted for NASH found to have evidence of NASH on graft biopsy after 6 months. Lower rates were reported by Yalamanchili et al., ${ }^{50}$ who performed a retrospective study from 1986 to 2004 of patients undergoing LT for NASH-related cirrhosis or cryptogenic cirrhosis. In the 227 patients undergoing follow-up liver biopsy, the calculated probability of developing hepatic steatosis was $8.2 \%, 13.6 \%$, $24.9 \%$ and $32.9 \%$ at $1,2,5$ and 10 years, respectively. Only $6 \%$ of these patients developed NASH. ${ }^{50}$ In a study by Contos et al. ${ }^{51}$ of patients with cirrhosis due to NASH undergoing LT, 3 of $27(11 \%)$ patients showed evidence of steatohepatitis. Consistent with other studies, serial biopsies demonstrated progression from steatosis to steatohepatitis. In their study, cumulative steroid exposure was also found to correlate with the development of NAFLD.

In contrast, El Atrache et al. ${ }^{52}$ found no statistically significant difference in steroid use, cholesterol and triglyceride levels, and BMI in patients with NASH recurrence compared to those without recurrence. In their retrospective, singlecenter study of all patients undergoing LT between 1996 
and 2008, they identified 46 patients with NASH and 37 patients with cryptogenic cirrhosis. Recurrence rates of NAFLD were not reported, but 20 patients showed pathological evidence of NASH with a mean follow-up time of 45.7 months. The mean time from LT until diagnosis of NASH was 18.2 months. Studies have also described the development of de novo NAFLD following LT. ${ }^{53}$

A retrospective study by Seo et al. ${ }^{54}$ looked at 68 patients transplanted at the University of California Davis, of which $84 \%$ were due to HCV. In that cohort, 12 patients (18\%) developed de novo NAFL and 6 patients ( $9 \%$ ) developed de novo NASH. Among LT patients with minimal donor steatosis, 8 patients $(12 \%)$ showed no significant change in steatosis and 17 patients $(25 \%)$ showed increased steatosis at postLT biopsy. An increase in BMI $>10 \%$ after LT was associated with development of de novo NAFLD (35\%; $p<0.05)$. In their study, differences among immunosuppressive regimens, such as prednisone, calcineurin inhibitors, and sirolimus, that may increase insulin resistance were not found to be statistically associated with de novo NAFLD.

On the other hand, a retrospective study of a single-center LT cohort found that steroid dosage after LT $(5.2 \pm 2.4 \mathrm{mg} /$ day vs. $7.1 \pm 4.7 \mathrm{mg} /$ day; $p=0.014$ ) was associated with de novo metabolic syndrome. ${ }^{55}$ In their cohort of 170 patients, de novo metabolic syndrome affected $32.9 \%$ of all patients, and was associated with NAFLD $(p=0.001)$. Further stratification of data found that NAFLD rates were higher among patients who developed metabolic syndrome within 1 year ( $23 \%$ vs. $50 \% ; p=0.001)$ or within 2 years $(25.4 \%$ vs. $45.8 \% ; p=0.015)$ after LT compared with patients without metabolic changes. In addition to metabolic syndrome risk factors contributing to recurrence of NAFLD, PNPLA3 genotype has also been associated with increased rates of recurrence. ${ }^{56}$

\section{Retransplantation for NASH}

Overall, there is a paucity in data regarding retransplantation for NASH. Despite the relatively high rates of recurrence of NAFLD and lower rates of recurrent NASH, studies with 5-10 year follow-up have not suggested increased rates of retransplantation. In the single-center study by El Atrache et al., ${ }^{52} 6$ patients with NASH recurrence $(30 \%)$ underwent retransplantation. Three of those were due to graft failure secondary to NASH recurrence, while the others were due to hepatic artery thrombosis $(n=2)$ and autoimmune hepatitis $(n=1)$. Longer-term studies are needed for further investigation.

\section{Conclusions}

NASH is currently the most rapidly growing indication for LT in the USA. Although the incidence of NASH-related cirrhosis as an indication for LT is increasing, wait-list mortality or removal is higher in NASH patients than for other etiologies. After LT, the overall survival for NASH patients is similar to survival rates for other etiologies, although some studies have noted increased postoperative mortality due to sepsis or cardiovascular complications. Studies of recurrence rates for NAFL and NASH following LT have demonstrated trends in risk factors contributing to recurrence that require close monitoring in posttransplant clinic for early diagnosis of recurrence and aggressive risk factor modification, including weight loss and management of T2DM and dyslipidemia. Further, large, prospective trials are warranted to reach consensus on identifying specific risk factors and developing effective strategies to prevent NASH recurrence.

\section{Conflict of interest}

The authors have no conflict of interests related to this publication.

\section{Author contributions}

Drafted the manuscript ( $A L, A G)$, provided critical revisions (DK, AAL, CG, GC, DK, AA), helped develop the theoretical framework and supervised the project (AA). All authors made substantial contributions to the conception of the work and helped contribute to the final version of the manuscript.

\section{References}

[1] Younossi ZM, Koenig AB, Abdelatif D, Fazel Y, Henry L, Wymer M. Global epidemiology of nonalcoholic fatty liver disease-Meta-analytic assessment of prevalence, incidence, and outcomes. Hepatology 2016;64:73-84. doi: $10.1002 /$ hep. 28431.

[2] Leite NC, Salles GF, Araujo AL, Villela-Nogueira CA, Cardoso CR. Prevalence and associated factors of non-alcoholic fatty liver disease in patients with type-2 diabetes mellitus. Liver Int 2009;29:113-119. doi: 10.1111/j. 1478-3231.2008.01718.x.

[3] Pais R, Barritt AS 4th, Calmus Y, Scatton O, Runge T, Lebray P, et al. NAFLD and liver transplantation: Current burden and expected challenges. J Hepatol 2016;65:1245-1257. doi: 10.1016/j.jhep.2016.07.033.

[4] Younossi ZM, Stepanova M, Afendy M, Fang Y, Younossi $Y$, Mir H, et al. Changes in the prevalence of the most common causes of chronic liver diseases in the United States from 1988 to 2008. Clin Gastroenterol Hepatol 2011;9:524-530.e1. doi: 10.1016/j.cgh.2011.03.020.

[5] Pappachan JM, Babu S, Krishnan B, Ravindran NC. Non-alcoholic fatty liver disease: A clinical update. J Clin Transl Hepatol 2017;5:384-393. doi: 10.14218/JCTH.2017.00013.

[6] Younes R, Bugianesi E. Should we undertake surveillance for HCC in patients with NAFLD? J Hepatol 2018;68:326-334. doi: 10.1016/j.jhep.2017.10.006.

[7] Chalasani N, Younossi Z, Lavine JE, Charlton M, Cusi K, Rinella M, et al. The diagnosis and management of nonalcoholic fatty liver disease: Practice guidance from the American Association for the Study of Liver Diseases. Hepatology 2018;67:328-357. doi: 10.1002/hep.29367.

[8] Day CP. From fat to inflammation. Gastroenterology 2006;130:207-210. doi: $10.1053 / j . g a s t r o .2005 .11 .017$.

[9] Musso G, Gambino R, De Michieli F, Cassader M, Rizzetto M, Durazzo M, et al. Dietary habits and their relations to insulin resistance and postprandial lipemia in nonalcoholic steatohepatitis. Hepatology 2003;37:909-916. doi: 10.1053/jhep.2003.50132.

[10] Cai D, Yuan M, Frantz DF, Melendez PA, Hansen L, Lee J, et al. Local and systemic insulin resistance resulting from hepatic activation of IKK-beta and NF-kappaB. Nat Med 2005;11:183-190. doi: 10.1038/nm1166.

[11] Arkan MC, Hevener AL, Greten FR, Maeda S, Li ZW, Long JM, et al. IKK-beta links inflammation to obesity-induced insulin resistance. Nat Med 2005;11: 191-198. doi: 10.1038/nm1185.

[12] Romeo S, Kozlitina J, Xing C, Pertsemlidis A, Cox D, Pennacchio LA, et al. Genetic variation in PNPLA3 confers susceptibility to nonalcoholic fatty liver disease. Nat Genet 2008;40:1461-1465. doi: 10.1038/ng.257.

[13] Kupcinskas J, Valantiene I, Varkalaite G, Steponaitiene R, Skieceviciene J, Sumskiene J, et al. PNPLA3 and RNF7 gene variants are associated with the risk of developing liver fibrosis and cirrhosis in an Eastern European population. J Gastrointestin Liver Dis 2017;26:37-43. doi: 10.15403/jgld.2014. 1121.261.pnp.

[14] Stender S, Kozlitina J, Nordestgaard BG, Tybjærg-Hansen A, Hobbs HH, Cohen JC. Adiposity amplifies the genetic risk of fatty liver disease conferred by multiple loci. Nat Genet 2017;49:842-847. doi: 10.1038/ng.3855.

[15] Vernon G, Baranova A, Younossi ZM. Systematic review: the epidemiology and natural history of non-alcoholic fatty liver disease and non-alcoholic steatohepatitis in adults. Aliment Pharmacol Ther 2011;34:274-285. doi: 10.1111/j.1365-2036.2011.04724.x.

[16] Hashimoto E, Tokushige K. Prevalence, gender, ethnic variations, and prognosis of NASH. J Gastroenterol 2011;46:63-69. doi: 10.1007/s00535-010-0311-8.

[17] Sayiner M, Koenig A, Henry L, Younossi ZM. Epidemiology of nonalcoholic fatty liver disease and nonalcoholic steatohepatitis in the United States and the rest of the world. Clin Liver Dis 2016;20:205-214. doi: 10.1016/j.cld. 2015.10.001. 
[18] Rinella ME. Nonalcoholic fatty liver disease: a systematic review. JAMA 2015: 313:2263-2273. doi: 10.1001/jama.2015.5370.

[19] Brunt EM, Kleiner DE, Wilson LA, Belt P, Neuschwander-Tetri BA. Nonalcoholic fatty liver disease (NAFLD) activity score and the histopathologic diagnosis in NAFLD: distinct clinicopathologic meanings. Hepatology 2011;53:810-820. doi: 10.1002/hep.24127.

[20] Kleiner DE, Brunt EM, Van Natta M, Behling C, Contos MJ, Cummings OW, et al. Design and validation of a histological scoring system for nonalcoholic fatty liver disease. Hepatology 2005;41:1313-1321. doi: 10.1002/hep.20701.

[21] Loomba R, Abraham M, Unalp A, Wilson L, Lavine J, Doo E, et al. Association between diabetes, family history of diabetes, and risk of nonalcoholic steatohepatitis and fibrosis. Hepatology 2012;56:943-951. doi: 10.1002/hep. 25772.

[22] Loomba R, Sanyal AJ. The global NAFLD epidemic. Nat Rev Gastroenterol Hepatol 2013;10:686-690. doi: 10.1038/nrgastro.2013.171

[23] Estes $\mathrm{C}$, Razavi $\mathrm{H}$, Loomba $\mathrm{R}$, Younossi Z, Sanyal AJ. Modeling the epidemic of nonalcoholic fatty liver disease demonstrates an exponential increase in burden of disease. Hepatology 2018;67:123-133. doi: 10.1002/hep.29466.

[24] Falck-Ytter Y, Younossi ZM, Marchesini G, McCullough AJ. Clinical features and natural history of nonalcoholic steatosis syndromes. Semin Liver Dis 2001;21:17-26. doi: 10.1055/s-2001-12926.

[25] Browning JD, Szczepaniak LS, Dobbins R, Nuremberg P, Horton JD, Cohen JC, et al. Prevalence of hepatic steatosis in an urban population in the United States: impact of ethnicity. Hepatology 2004;40:1387-1395. doi: 10.1002/ hep. 20466.

[26] Day CP. The potential role of genes in nonalcoholic fatty liver disease. Clin Liver Dis 2004;8:673-691. doi: 10.1016/j.cld.2004.04.001.

[27] Pan JJ, Fallon MB. Gender and racial differences in nonalcoholic fatty liver disease. World J Hepatol 2014;6:274-283. doi: 10.4254/wjh.v6.i5.274.

[28] Ogden CL, Carroll MD, Kit BK, Flegal KM. Prevalence of childhood and adult obesity in the United States, 2011-2012. JAMA 2014;311:806-814. doi: 10. 1001/jama.2014.732.

[29] Doycheva I, Issa D, Watt KD, Lopez R, Rifai G, Alkhouri N. Nonalcoholic steatohepatitis is the most rapidly increasing indication for liver transplantation in young adults in the United States. J Clin Gastroenterol 2018;52: 339-346. doi: 10.1097/MCG.0000000000000925.

[30] Wong RJ, Aguilar M, Cheung R, Perumpail RB, Harrison SA, Younossi ZM, et al. Nonalcoholic steatohepatitis is the second leading etiology of liver disease among adults awaiting liver transplantation in the United States. Gastroenterology 2015;148:547-555. doi: 10.1053/j.gastro.2014.11.039.

[31] Cholankeril G, Wong RJ, Hu M, Perumpail RB, Yoo ER, Puri P, et al. Liver transplantation for nonalcoholic steatohepatitis in the US: Temporal trends and outcomes. Dig Dis Sci 2017;62:2915-2922. doi: 10.1007/s10620-0174684-x.

[32] Parikh ND, Marrero WJ, Wang J, Steuer J, Tapper EB, Konerman M, et al. Projected increase in obesity and non-alcoholic-steatohepatitis-related liver transplantation waitlist additions in the United States. Hepatology 2017. doi: 10.1002/hep.29473.

[33] Charlton MR, Burns JM, Pedersen RA, Watt KD, Heimbach JK, Dierkhising RA. Frequency and outcomes of liver transplantation for nonalcoholic steatohepatitis in the United States. Gastroenterology 2011;141:1249-1253. doi: 10.1053/j.gastro.2011.06.061.

[34] Patel YA, Berg CL, Moylan CA. Nonalcoholic fatty liver disease: Key considerations before and after liver transplantation. Dig Dis Sci 2016;61:14061416. doi: 10.1007/s10620-016-4035-3.

[35] Goldberg D, Ditah IC, Saeian K, Lalehzari M, Aronsohn A, Gorospe EC, et al. Changes in the prevalence of hepatitis $C$ virus infection, nonalcoholic steatohepatitis, and alcoholic liver disease among patients with cirrhosis or liver failure on the waitlist for liver transplantation. Gastroenterology 2017;152: 1090-1099.e1. doi: 10.1053/j.gastro.2017.01.003.

[36] Kardashian AA, Dodge JL, Roberts J, Brandman D. Weighing the risks: Morbid obesity and diabetes are associated with increased risk of death on the liver transplant waiting list. Liver Int 2018;38:553-563. doi: 10.1111/liv.13523.

[37] Stine JG, Argo CK, Pelletier SJ, Maluf DG, Caldwell SH, Northup PG. Advanced non-alcoholic steatohepatitis cirrhosis: A high-risk population for pre-liver transplant portal vein thrombosis. World J Hepatol 2017;9:139-146. doi: 10.4254/wjh.v9.i3.139.
[38] Afzali A, Berry K, Ioannou GN. Excellent posttransplant survival for patients with nonalcoholic steatohepatitis in the United States. Liver Transpl 2012; 18:29-37. doi: 10.1002/It.22435.

[39] van den Berg EH, Douwes RM, de Meijer VE, Schreuder TCMA, Blokzijl H. Liver transplantation for NASH cirrhosis is not performed at the expense of major post-operative morbidity. Dig Liver Dis 2018;50:68-75. doi: 10. 1016/j.dld.2017.08.022.

[40] Malik SM, deVera ME, Fontes $\mathrm{P}$, Shaikh $\mathrm{O}$, Ahmad J. Outcome after liver transplantation for NASH cirrhosis. Am J Transplant 2009;9:782-793. doi: 10. $1111 /$ j.1600-6143.2009.02590.x.

[41] Wang X, Li J, Riaz DR, Shi G, Liu C, Dai Y. Outcomes of liver transplantation for nonalcoholic steatohepatitis: a systematic review and meta-analysis. Clin Gastroenterol Hepatol 2014;12:394-402.e1. doi: 10.1016/j.cgh.2013.09.023.

[42] Volk ML, Hernandez JC, Lok AS, Marrero JA. Modified Charlson comorbidity index for predicting survival after liver transplantation. Liver Transpl 2007; 13:1515-1520. doi: 10.1002/lt.21172.

[43] John PR, Thuluvath PJ. Outcome of liver transplantation in patients with diabetes mellitus: a case-control study. Hepatology 2001;34:889-895. doi: 10 . 1053/jhep.2001.29134.

[44] Guckelberger O, Mutzke F, Glanemann M, Neumann UP, Jonas S, Neuhaus R, et al. Validation of cardiovascular risk scores in a liver transplant population. Liver Transpl 2006;12:394-401. doi: 10.1002/It. 20722.

[45] Molloy RM, Komorowski R, Varma RR. Recurrent nonalcoholic steatohepatitis and cirrhosis after liver transplantation. Liver Transpl Surg 1997;3:177-178. doi: 10.1002/It.500030212.

[46] Everhart JE, Lombardero M, Lake JR, Wiesner RH, Zetterman RK, Hoofnagle $\mathrm{JH}$. Weight change and obesity after liver transplantation: incidence and risk factors. Liver Transpl Surg 1998;4:285-296. doi: 10.1002/It.500040402.

[47] Contos MJ, Luketic VA, Sterling RK, Shiffman ML, Mills S, Fisher RA, et al. The natural history of nonalcoholic steatohepatitis (NASH) following liver transplantation. Gastroenterology 2000;118:A1019. doi: 10.1016/S0016-5085 (00)86232-0.

[48] Bhati C, Idowu MO, Sanyal AJ, Rivera M, Driscoll C, Stravitz RT, et al. Longterm outcomes in patients undergoing liver transplantation for nonalcoholic steatohepatitis-related cirrhosis. Transplantation 2017;101:1867-1874. doi: 10.1097/TP.0000000000001709.

[49] Bhagat V, Mindikoglu AL, Nudo CG, Schiff ER, Tzakis A, Regev A. Outcomes of liver transplantation in patients with cirrhosis due to nonalcoholic steatohepatitis versus patients with cirrhosis due to alcoholic liver disease. Liver Transpl 2009;15:1814-1820. doi: 10.1002/It.21927.

[50] Yalamanchili K, Saadeh S, Klintmalm GB, Jennings LW, Davis GL. Nonalcoholic fatty liver disease after liver transplantation for cryptogenic cirrhosis or nonalcoholic fatty liver disease. Liver Transpl 2010;16:431-439. doi: 10. $1002 /$ It. 22004.

[51] Contos MJ, Cales W, Sterling RK, Luketic VA, Shiffman ML, Mills AS, et al. Development of nonalcoholic fatty liver disease after orthotopic liver transplantation for cryptogenic cirrhosis. Liver Transpl 2001;7:363-373. doi: 10. 1053/jlts.2001.23011.

[52] El Atrache MM, Abouljoud MS, Divine G, Yoshida A, Kim DY, Kazimi MM, et al. Recurrence of non-alcoholic steatohepatitis and cryptogenic cirrhosis following orthotopic liver transplantation in the context of the metabolic syndrome. Clin Transplant 2012;26:E505-E512. doi: 10.1111/ctr.12014.

[53] Morales E, Garcia R, Saksena S, Hubscher S, Elias E. Recurrrent and de novo non-alcoholic steatohepatitis (NASH) following orthotopic liver transplantation (OLT). J Hepatol 2000;32:57. doi: 10.1016/S0168-8278(00)80552-8.

[54] Seo S, Maganti K, Khehra M, Ramsamooj R, Tsodikov A, Bowlus C, et al. De novo nonalcoholic fatty liver disease after liver transplantation. Liver Transpl 2007;13:844-847. doi: 10.1002/It.20932.

[55] Sprinzl MF, Weinmann A, Lohse N, Tönissen H, Koch S, Schattenberg J, et al. Metabolic syndrome and its association with fatty liver disease after orthotopic liver transplantation. Transpl Int 2013;26:67-74. doi: 10.1111/j.14322277.2012.01576.x

[56] Finkenstedt A, Auer C, Glodny B, Posch U, Steitzer H, Lanzer G, et al. Patatinlike phospholipase domain-containing protein 3 rs738409-G in recipients of liver transplants is a risk factor for graft steatosis. Clin Gastroenterol Hepatol 2013;11:1667-1672. doi: 10.1016/j.cgh.2013.06.025. 